\title{
Complex interplay of short- and long-chain branching on thermal and rheological properties of ethylene/a- olefin copolymers made by metallocene catalysts with oscillating ligand structure
}

Mostafa Ahmadi ${ }^{*}$, Faezeh Rezaei ${ }^{2}$, Seyed Mohammad Mahdi Mortazavi ${ }^{3}$, Mehdi Entezam ${ }^{4}$ Florian J. Stadler ${ }^{5 *}$ 


\section{Abstract}

Unbridged oscillatory metallocenes are prone to produce heterogeneous chain microstructure, for instance, giving rise to elastomeric polypropylene. Here, we use the bis(2phenylindenyl) $\mathrm{ZrCl}_{2} / \mathrm{MAO}$ catalyst system, featuring oscillating ligands, to copolymerize ethylene with several $\alpha$-olefins. Through investigation of chain microstructure, thermal and rheological properties, we show that the complex behavior of these materials can be justified by considering a catalyst with dual active center having different comonomer affinities. This behavior is consistent with the proposed mechanism of fast oscillating ligands, becoming locked upon proximity of cocatalyst counteranion, leading to diverse stereo- and comonomer selectivity. The unlocked, oscillating active center with lower comonomer response gives rise to mostly linear chains and is not affected by the presence of comonomer, while the locked one creates long chain branched chains, whose frequency decreases by introduction of comonomer. Copolymer samples exhibited bimodal MWD, dual crystallization mechanisms, and low frequency plateau modulus, specifically at higher comonomer levels. It was concluded that the overall behavior is determined by complex interplay of SCB and LCB, which leads to thermorheological complexity as well as phase separation.

\section{Introduction}

The catalytic copolymerization of ethylene with $\alpha$-olefins is a typical way of introducing shortchain branching (SCB) in the polymer molecules and, thereby, causing significant changes in the physical polymer properties, such as melting and glass transition temperatures, mechanical, and optical properties, all of which define the type and useful range of their application [1-3]. New generations of catalysts and technologies are leading to new families of polyethylenes due to the ability of these systems to introduce controlled amounts of $\alpha$-olefins into polyethylene with multifarious distribution patterns [4-9]. 
Within the commercially available catalysts, metallocenes have shown major advantages over traditional Ziegler-Natta and chromium catalysts due to their higher degrees of control over broad microstructure factors. Several structural parameters can be adjusted to tune the specificity of metallocenes to produce favored chains with specific microstructures [9]. Metallocenes with less hindered metal centers have a higher tendency to incorporate bulkier $\alpha$-olefin comonomers. Moreover, polymer chains containing a terminal vinyl group, created in situ, can be also incorporated into the growing chains, leading to the formation of long-chain branched (LCB) polymers. With higher affinity to comonomer incorporation comes better chances of LCB formation $[10,11]$. Narrow molecular weight distribution made by metallocenes leads to enhanced mechanical properties, but decreases melt processability. Long-chain branches are known to strongly improve the processing behavior of polymers by not only increasing the shear-thinning behavior at smaller angular frequencies but also improving the stability of elongation-dominated processes by strain hardening $[12,13]$.

LCBs are hard to be distinguished by traditional characterization methods such as NMR, instead, they can cause significant deviation in rheological properties from their linear counterparts, namely, the zero-shear rate viscosity, terminal relaxation time and temperature dependency of rheological parameters are considerably altered [14-16]. Many researchers have, therefore, used these rheological fingerprints to assess the microstructure and to determine the LCB-content and topography $[14,17-19]$.

Metallocenes with high comonomer selectivity can produce elastomeric ethylene/ $\alpha$-olefin copolymers with lower densities and crystallinities compared to the corresponding thermoplastics [20]. Physical properties of these olefinic elastomers are in some cases competitive to conventional elastomers and the efficiency of polyolefin production and processing allows them to be produced at a competitive cost/performance ratio. One of the interesting groups of metallocenes with high comonomer affinity are conformationally dynamic unbridged metallocenes based on indenyl ligands 
[21]. Ligand oscillation between different coordination geometries during polymerization of propylene can produce atactic-isotactic stereoblock polypropylene [21]. The structures of these materials differ from those made by heterogeneous catalysts or ansa-metallocenes, which consequently also leads to different properties; e. g. these materials exhibit broad melting peaks and high-melting points typical of the materials produced from heterogeneous catalysts, but possess molecular weight distributions slightly broader than typical ansa-metallocene polymers [22]. Several factors including feed composition, reaction temperature, type of cocatalyst and even the polarity of the solvent are involved in the heterogeneity of the chain structures made by these catalysts [20-25].

Bis(2-arylindenyl) metallocene catalysts are surprisingly effective at incorporating $\alpha$-olefins, even far superior than unsubstituted bis(indenyl) metallocenes [1]. They have shown a remarkably heterogeneous composition distribution as confirmed by thermal properties, GPC and NMR measurements [1, 21-23]. However, to the best of our knowledge, the effects of this complex microstructure on the rheological properties have not been investigated so far.

In this work, we have used bis(2-phenylindenyl) $\mathrm{ZCl}_{2}$ catalysts for copolymerization of ethylene with several $\alpha$-olefins and shown that the significant effect of LCB formation on the rheological properties is extensively convoluted with the effect of chemical composition heterogeneity. The paper is organized as follows: After introducing the materials and characterization methods, thermal properties are investigated in depth. Then different aspects of rheological properties including small angle oscillatory shear and creep data are discussed and final conclusions are drawn based on the mechanism of oscillation and locking in this class of metallocene catalysts.

\section{Experimental}

\subsection{Samples}


Ethylene copolymerization reactions with propylene, 1-hexene and 1-octene were carried out in toluene using bis(2-phenylindenyl) $\mathrm{ZrCl}_{2}$ as the catalyst, and methylaluminoxane (MAO) as the cocatalyst. Toluene $(450 \mathrm{ml})$ was introduced in a $1 \mathrm{~L}$ Büchi reactor and dissolved air was removed by several times saturation and evacuation by nitrogen followed by several round of similar cycles using ethylene gas. Comonomer, MAO ([Al]/[Zr]=4000:1) and catalyst solution $(1 \mu \mathrm{mol})$ were introduced respectively, and reaction temperature and pressure was increased to the set points (60 ${ }^{\circ} \mathrm{C}$ and 4 bar, respectively) [2, 3]. The propylene comonomer was introduced continuously as determined in Table 1, but the liquid 1-hexene and 1-octene comonomers were added batch-wise in the beginning of polymerizations as mentioned. The final comonomer incorporation compared to the feed composition was lower than $20 \%$ in all experiments, therefore, significant composition drift could be ruled out. The relevant polymerization conditions and sample definitions are depicted in Table 1.

\subsection{Characterization}

Molecular weight distribution (MWD) of polymers were determined by a PL-210 gel permeation chromatograph (Polymer Laboratories, UK) using 1,2,4-trichlorobenzene as solvent at $140{ }^{\circ} \mathrm{C}$.

Differential scanning calorimetry (DSC) (Mettler-Toledo, $823^{\mathrm{e}}$ Module) was used for determination of crystallization and melting behavior of synthesized polymers. Samples were heated from room temperature to $160{ }^{\circ} \mathrm{C}$ at a rate of $10{ }^{\circ} \mathrm{C} / \mathrm{min}$, and remained for 10 minutes at this temperature, followed by cooling to $25^{\circ} \mathrm{C}$ at the same rate. Finally, samples were heated from $25{ }^{\circ} \mathrm{C}$ to $160{ }^{\circ} \mathrm{C}$ at the same heating rate and final endothermic melting peaks were collected at the last heating cycle.

The flash-DSC developed by Mettler Toledo Switzerland, was used to capture the fast thermal transitions usually encountered in polyolefins that are not simply trackable by normal DSC. It uses a 
highly sensitive sensor that allows isothermal experiments for times as short as $0.001 \mathrm{~s}$ and can perform heating and cooling cycles with rates as high as $\pm 10000 \mathrm{~K} / \mathrm{s}$. A sample of approximately 20 ng was placed on the sensor chip and a good thermal contact to the surface was established by performing one heating/cooling cycle. Details of measurement conditions are explained in Section 3.1 .

Linear viscoelastic properties were characterized by small amplitude oscillatory shear (SAOS) experiment using Anton Paar's MCR 301 rheometer at strain control configuration by $25 \mathrm{~mm}$ parallel plate tools and $1 \mathrm{~mm}$ gap. All samples were stabilized with $0.5 \mathrm{wt} \%$ of Irganox 1010 and 0.5 wt\% Irgafos 168 and all measurements were performed under nitrogen atmosphere $[13,15]$. Disk samples of about $1.1 \mathrm{~mm}$ thickness were prepared by compression molding at $190{ }^{\circ} \mathrm{C}$. Dynamic strain sweeps were performed at frequency of $1 \mathrm{rad} / \mathrm{s}$ in advance to identify the linear viscoelastic regime, and all measurements were done using strain amplitude of $1 \%$ to $5 \%$, in the linear domain, correspondingly. Dynamic frequency sweep measurements were carried out at three temperatures of 160,190 , and $220{ }^{\circ} \mathrm{C}$ and angular frequency range of 100 to $0.01 \mathrm{rad} / \mathrm{s}$. Measurements were repeated on a different rheometer (ARES rheometer of TA instruments) to check the reliability of the data and to exclude degradation from the possible reasons of the observed complex rheological behavior and all measurements were nicely superimposable.

Creep measurements were performed at $190{ }^{\circ} \mathrm{C}$ at constant shear stress corresponding to the one tenth of the shear stress reported in the frequency sweep test at a frequency of $0.1 \mathrm{rad} / \mathrm{s}$. This value was experimentally found suitable so that it was small enough to avoid the nonlinear regime and large enough to avoid noisy data. 


\section{Results and Discussion}

Unbridged metallocene catalysts have conformationally oscillating ligands and if ligands are not symmetric, this can lead to diverse microstructures made at different conformations. In particular, chains with different comonomer contents may be formed in copolymerization reactions, if different conformations show dissimilar comonomer selectivity. Since the comonomer incorporation is proved to affect the extent of LCB formation, this could also lead to heterogeneity of LCB distribution [13]. We have, therefore, used thermal and rheological properties to track such alterations, since the former is extensively dependent on SCB content and the latter is profoundly sensitive to the LCB structure $[2,3,26-28]$.

\subsection{Chemical composition heterogeneity as depicted in thermal properties}

Ethylene was copolymerized with propylene at two different levels, and with 1-hexene and 1octene at three levels. The resultant weight average molecular weight and polydispersity indices, measured by GPC, are shown in Table 1 and the MWD curves are compared in Figure 1. Data reported in Table 1 shows that the average molecular weight increases upon introduction of comonomers, but it decreases to lower values as comonomer concentration is further increased. The initial upturn of molar mass can be attributed to the so called "comonomer effect" where the increase in overall propagation rate constant can lead to longer chains [29]. This effect explains why ethylene with the highest homopolymerization rate among all olefins shows even higher polymerization rates in presence of small amounts of $\alpha$-olefin comonomers $[29,30]$. However, comonomer can decrease the molecular weight by increasing transfer reactions, which dominates the behavior at higher concentrations [29-31].

The extensive asymmetry of MWD curves (Figure 1) proves enhanced diversity in chain length at higher comonomer levels. In particular, there is a distinct shoulder on the high molecular weight tail of C6-6 and C8-6, with highest comonomer ratios. As metallocenes with defined structure are 
supposed to exhibit single-site behavior, finding a high molecular weight tail reveals the presence of at least two active centers with dissimilar response to comonomer. One is less affected by the presence of comonomer, producing approximately the same chain length as in the absence of comonomer and the other has high comonomer response, making shorter chains upon introduction of comonomer.

Table 1: Characteristics of ethylene/ $\alpha$-olefin copolymers

\begin{tabular}{|c|c|c|c|c|c|c|c|c|c|}
\hline Sample & $\begin{array}{l}\text { Comonomer } \\
\text { type }\end{array}$ & $\begin{array}{l}\text { Comonomer } \\
\text { in Feed } \\
(\mathrm{mmol} / \mathrm{L})\end{array}$ & $\begin{array}{l}\text { Comonomer in } \\
\text { copolymer } \\
(\mathrm{wt} \%)^{\mathrm{b}}\end{array}$ & $\begin{array}{l}\mathrm{M}_{\mathrm{W}} \\
(\mathrm{kg} / \mathrm{mol})\end{array}$ & PDI & $\begin{array}{l}\mathrm{T}_{\mathrm{m}} \\
\left({ }^{\circ} \mathrm{C}\right)\end{array}$ & $\begin{array}{l}\mathrm{T}_{\mathrm{c}} \\
\left({ }^{\circ} \mathrm{C}\right)\end{array}$ & $\begin{array}{l}X_{c} \\
(\%)\end{array}$ & $\begin{array}{l}\text { Density } \\
(\mathrm{g} / \mathrm{ml})\end{array}$ \\
\hline Homo & - & 0 & 0 & 195 & 3.7 & 137.3 & 115.2 & 80.1 & 0.96 \\
\hline C3-1 & \multirow[t]{2}{*}{ Propylene } & $0.05^{\mathrm{a}}$ & n.d, & 328 & 7.7 & 133.3 & 112.0 & 63.5 & n.d. \\
\hline C3-2 & & $0.10^{\mathrm{a}}$ & 4.0 & 66 & 4.7 & 122.9 & 108.2 & 59.7 & 0.92 \\
\hline C6-1 & \multirow[t]{3}{*}{ 1-hexene } & 35 & 2.8 & 187 & 3.0 & 126.7 & 106.5 & 43.2 & 0.93 \\
\hline C6-3 & & 106 & 4.1 & 248 & 4.1 & 115.6 & 100.6 & 38.6 & 0.91 \\
\hline C6-6 & & 212 & 7.1 & 68 & 9.0 & 114.0 & 103.0 & 40.2 & 0.91 \\
\hline C8-1 & \multirow[t]{3}{*}{ 1-octene } & 35 & 2.7 & 351 & 3.1 & 129.1 & 109.3 & 43.1 & 0.93 \\
\hline C8-3 & & 106 & 4.6 & 93 & 4.2 & 120.7 & 108.1 & 40.8 & 0.93 \\
\hline C8-6 & & 212 & 8.0 & 40 & 4.8 & 112.3 & 100.9 & 38.7 & 0.92 \\
\hline
\end{tabular}

a: relative flow rate of propylene to ethylene. b: Calculated based on ${ }^{13} \mathrm{C}-\mathrm{NMR}$ data given in Ref [3]. n.d. not determined

Regrettably, GPC-MALLS was not available to determine the molar mass distribution along with the branching distribution. Based on our previous experience with LCB-mPE, $\mathrm{M}_{\mathrm{n}}$ should be correct or only slightly underestimated, while the measured value of $\mathrm{M}_{\mathrm{w}}$ should be distinctly too low, especially for materials with a broader molar mass distribution such as the ones in this article. The long-chain branching frequency decreases at higher comonomer concentrations for catalysts containing single active center [13]. However, the nature of the oscillating metallocene catalyst basically means that the steric hindrance of the active center varies, which, consequently means that both the comonomer incorporation levels of the two active center types and their long-chain branch incorporation varies. 

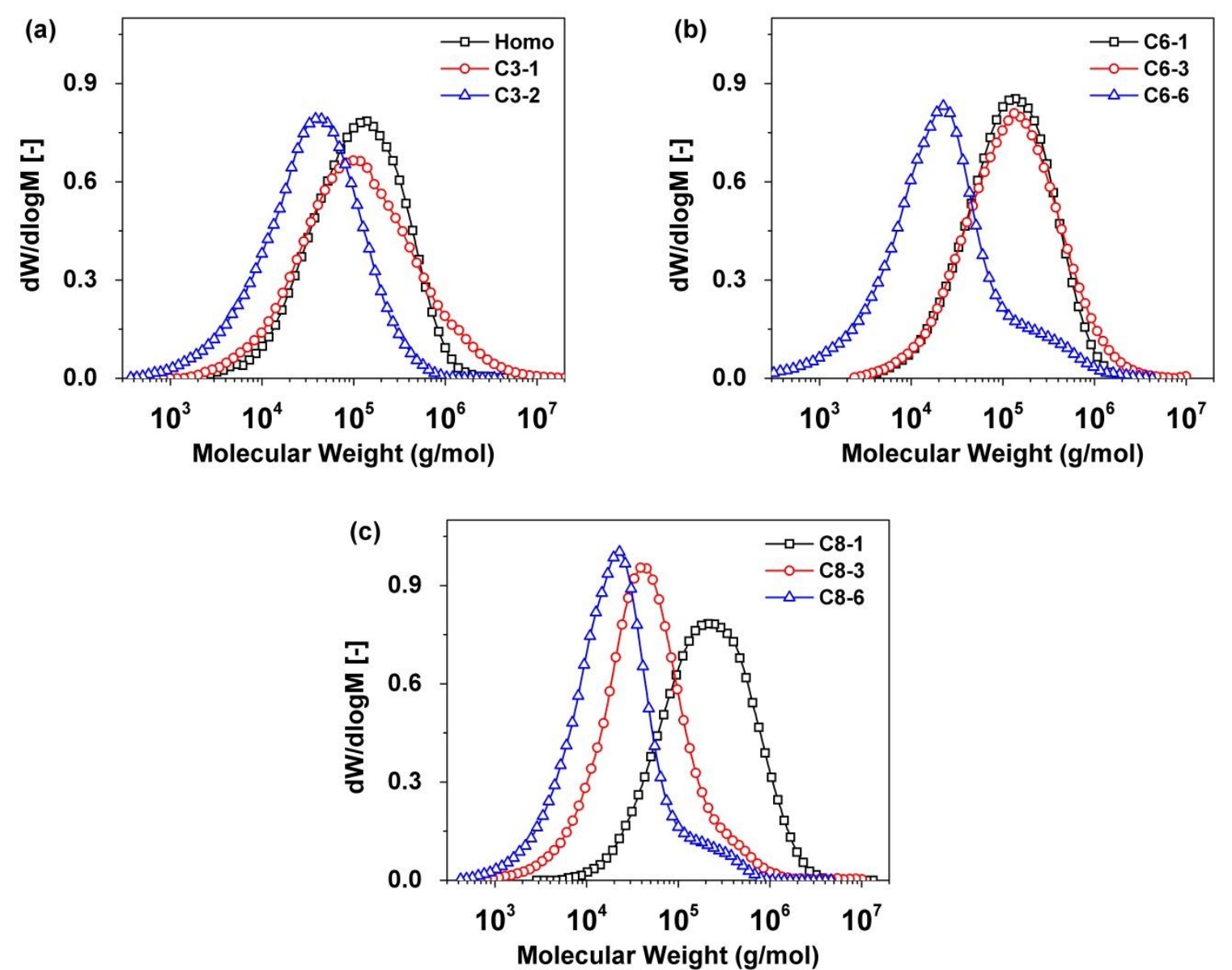

Figure 1. Molecular weight distribution of ethylene/a-olefin copolymers: (a) homopolymer and C3 (b) C6 and (c) C8 sample series.

The diverse comonomer affinities should be more evident in thermal behavior. Melting $\left(\mathrm{T}_{\mathrm{m}}\right)$ and crystallization $\left(\mathrm{T}_{\mathrm{c}}\right)$ temperatures are dictated by the length of ethylene sequence and are therefore affected by the introduction of comonomer $[2,3]$. The crystallization and melting peaks of the three synthesized sets of samples are compared in Figure 2 and the corresponding $T_{c}$ and $T_{m}$ values are listed in Table 1. As expected, both $\mathrm{T}_{\mathrm{c}}$ and $\mathrm{T}_{\mathrm{m}}$ decrease by the introduction of comonomer and this effect is more noticeable when using bulkier comonomers [32].

The secondary crystallization peaks, as depicted in the inset plots of Figure 2, appear at temperatures between 55 to $80{ }^{\circ} \mathrm{C}$. These peaks become more distinguishable and shift towards lower temperature values, using bulkier comonomer at higher levels, likewise. The primary 
crystallization process includes faster folding of longer ethylene sequences, having a higher melting point, into stacked lamellae that form spherulite from the free melt. Crystallinity may further increase due to secondary crystallization of the shorter ethylene sequences that form thinner lamellae in the amorphous regions between the primary lamellae inside the spherulite [33].
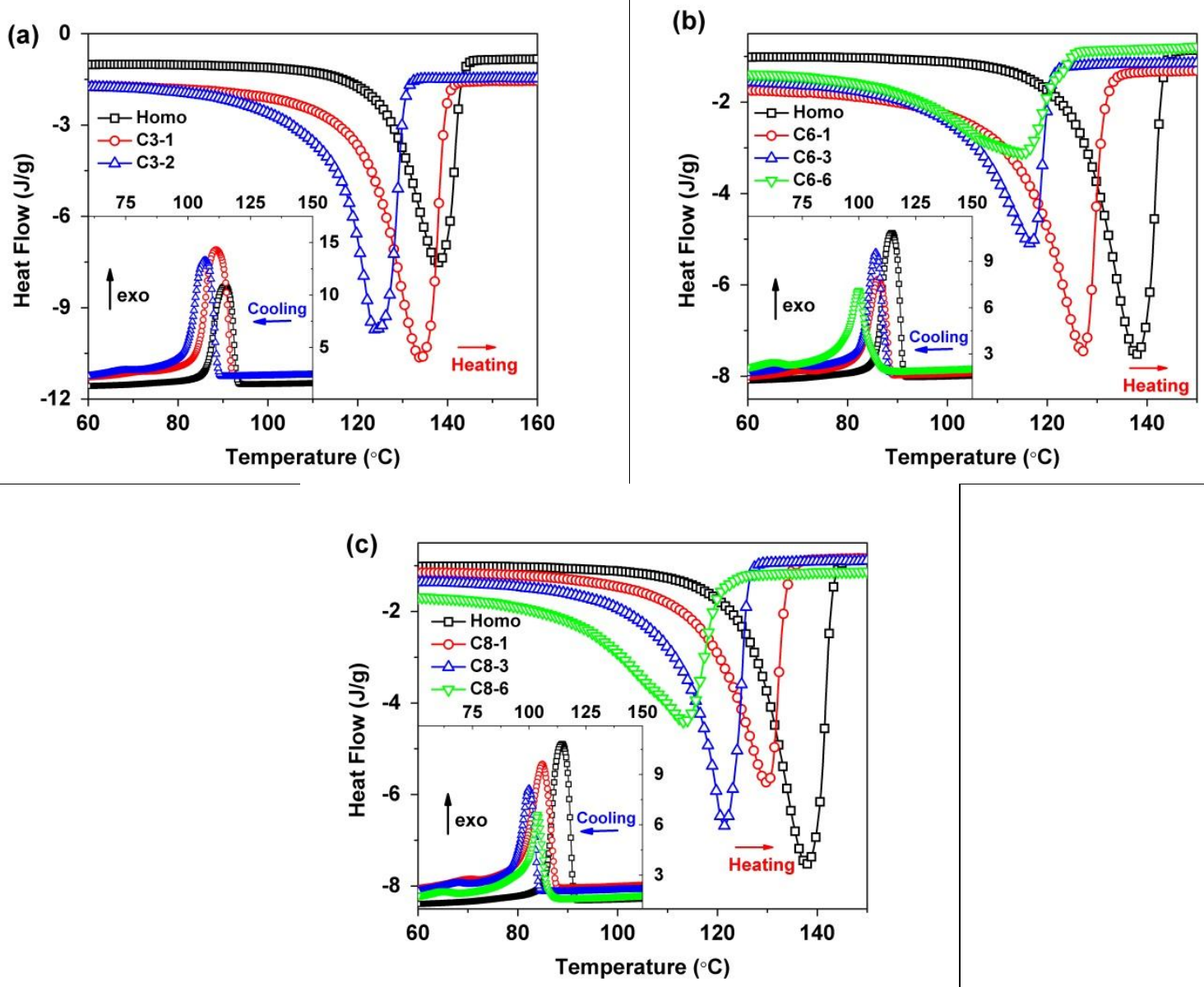

Figure 2. Crystallization (main) and melting (inset) peaks of the samples made by copolymerization of ethylene with (a) propylene (series C3) (b) 1-hexene (series C6) and (c) 1-octene (series C8). The main and inset plots have similar units.

Both the melting and crystallization peaks become broader upon introduction of comonomer. This is expected, as the intermolecular ethylene sequence length distribution follows a Flory distribution function and, therefore, should be rather broad [34]. Ethylene sequences are folded to make lamellae and side branches of olefinic comonomers are excluded from crystal cells. As lamellae are melted according to their thickness, the broadness of melting temperature peak is a 
direct signature of ethylene sequence length distribution [35]. However, the melting peaks of C6 and C8 copolymers at highest comonomer fractions are so broad that a low temperature shoulder (around $105{ }^{\circ} \mathrm{C}$ ) can be distinguished and essentially a second peak can be expected at lower temperatures. This is another reliable indication for the presence of two types of active centers with significantly dissimilar comonomer selectivity, consistent to MWD results. It is worth noting that the crystallization ability decreases significantly at lower temperatures and shorter ethylene sequences, and therefore the perceptible shoulder at this region represents considerable fraction of chains with high comonomer contents [32].

The conventional DSC is unable to capture the fast thermal transitions of polyolefins; therefore, we used flash-DSC to track the fast transitions in Sample C8-6 (with highest concentration of bulkiest comonomer). After removing the thermal history at $160{ }^{\circ} \mathrm{C}$, different cooling and heating rates were applied. The sample was crystallized using different cooling rates of $-10,-100$ and -1000 $\mathrm{K} / \mathrm{s}$ followed by heating at the rate of $1000 \mathrm{~K} / \mathrm{s}$ and the corresponding peaks are depicted in Figure 3a. The crystallization peak shifts to lower temperatures as the cooling rate increases, as expected (inset plot). Interestingly enough, a low-temperature melting peak becomes noticeable after fastest cooling rate (main plot). Accordingly, the fast crystallization rate of $1000 \mathrm{~K} / \mathrm{s}$ has avoided rearrangement and cocrystallization of different ethylene sequence lengths. Using this cooling rate, we performed $1 \mathrm{~s}$ annealing step at temperatures between 70 and $100{ }^{\circ} \mathrm{C}$ during cooling ramp from 160 to $0{ }^{\circ} \mathrm{C}$, and the consequent melting peaks are depicted in Figure $3 \mathrm{~b}$. 

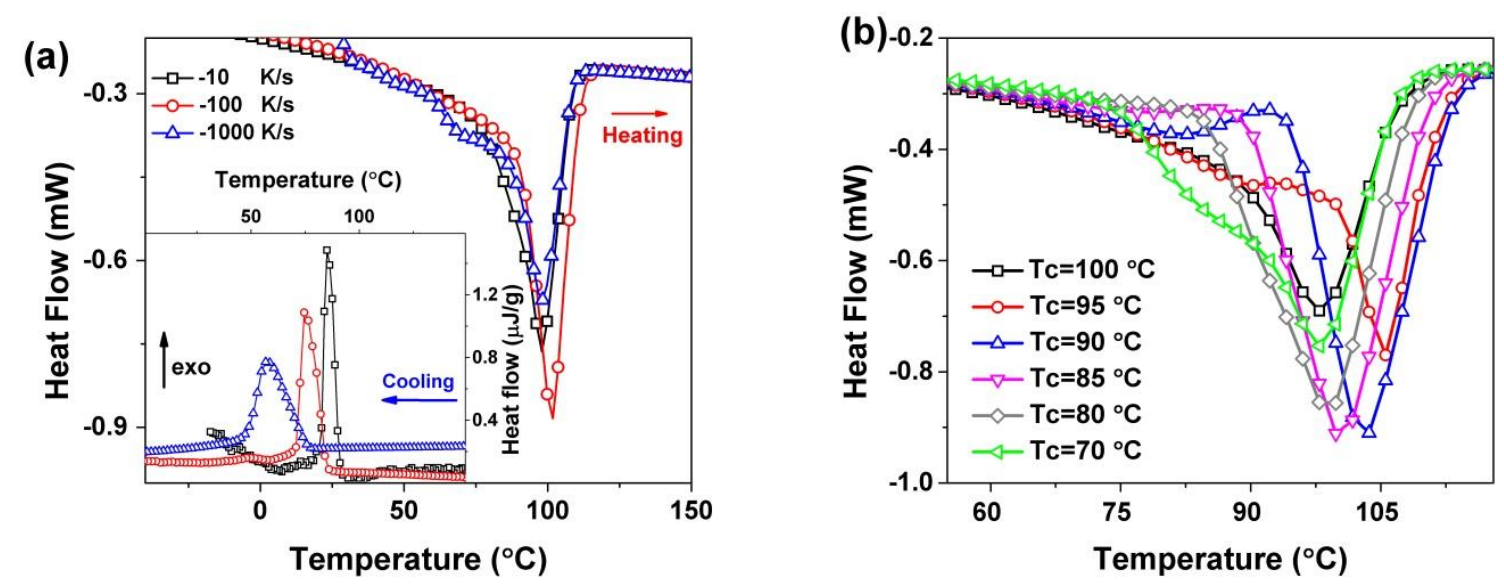

Figure 3. Flash-DSC curves of Sample C8-6: (a) crystallization peaks (inset) at cooling rates shown in the legend and subsequent melting peaks at a heating rate of $1000 \mathrm{~K} / \mathrm{s}$ (main). (b) Melting peaks at cooling rate of 1000 $\mathrm{K} / \mathrm{s}$ after 1 second isothermal annealing, at temperatures (Tc) indicated in the legend, during crystallization at a rate of $1000 \mathrm{~K} / \mathrm{s}$.

The bimodal melting peak is apparent for several annealing temperatures, which confirms the presence of bimodal comonomer distribution. It seems that isothermal crystallization temperature of $100{ }^{\circ} \mathrm{C}$ is too high even for formation of thick lamellae, and both crystals are formed and cocrystallized during the consequent cooling ramp. However, annealing at lower temperatures leads to two separate melting peaks. All in all, flash-DSC can prove the presence of bimodal comonomer distribution that can be depicted in both crystallization and melting peaks upon special thermal treatments. This is the particular specificity of oscillating metallocenes with asymmetric ligands that to the best of our knowledge was not explored, using thermal properties.

\subsection{LCB heterogeneity as depicted in rheological properties}

A qualitative distinction between various polymers can be obtained from the finding whether a polymer melt is thermorheologically simple or complex. If the relaxation phenomena involved in dynamics of a polymer sample over broad frequency domain follow the same temperature dependencies, a master curve can be built by shifting dynamic moduli using single activation energy $\left(E_{a}\right)$. Despite the fact that both SCB and LCB increase the activation energy still $E_{a}$ is independent 
of the phase angle $\delta[13,15,16,36]$. While the highly branched low density polyethylene (LDPE) is only slightly thermorheologically complex, for the long-chain branched metallocene catalyzed polyethylenes a significant dependence of the activation energy on the relaxation time is detected $[13,15]$. At shorter relaxation times, $\mathrm{E}_{\mathrm{a}}$ is very close to that of linear polyethylenes, but it increases at longer relaxation times. The lower $\mathrm{E}_{\mathrm{a}}$ values are assumed to be due to linear molecules and the higher ones to long-chain branched molecules. It has been concluded for metallocene catalyzed polyethylenes that the thermorheological complexity is due to the presence of a mixture of linear and long-chain branched chains, with different relaxation mechanisms and temperature dependencies [15]. Similar trend in confirmed for linear/branched polyethylene blends [28, 37]. The population of LCB and linear chains should be a characteristic of the investigated catalyst, depending on the frequency of creating double bond ended chains and incorporating them into the growing chains. For the oscillatory catalysts with two catalyst conformations higher diversity of microstructure is expected.

The van Gurp-Palmen (vGP) is a plot of phase angle versus complex modulus which is extensively used for detecting thermorheological complexity $[13,15,36,37]$. In case of thermorheological simplicity, all frequency sweep measurements at different temperatures should superimpose. However, deviations are reported for metallocene catalyzed polyethylene and blend of linear and branched chains [15, 28, 37]. Figure 4 (and Figure SI1 in the Supporting Information) shows the vGP plots of the homopolymer and three sets of copolymers measured at 160, 190 and $220{ }^{\circ} \mathrm{C}$, along with a purely linear polyethylene homopolymer adopted from literature, having $\overline{M w}$ of $173 \mathrm{~kg} / \mathrm{mol}$ and PDI of $2[13,15,16,18]$. Thermorheological complexity is evident especially for the homopolymer sample. Samples tend to behave thermorheologically simpler with increasing comonomer content in the feed, which confirms decrease of LCB formation and predominant formation of linear chains [13]. Surprisingly, for ethylene/propylene and ethylene/1-hexene copolymers at highest comonomer levels, i.e. C3-2 and C6-6 samples, despite thermorheological 
simplicity, there is a second drop in phase angle and the final relaxation corresponding to the phase angle of $90^{\circ}$ could not be achieved at lower frequencies. The second drop in the phase angle at lower modulus values in high-comonomer content copolymers could be attributed to the phase separation between chains with different comonomer contents, made at different catalyst conformations. Due to similar modulus and solubility of two comonomer poor and comonomer rich phases, such a phase separation is very hard to detect experimentally using Atomic Force Microscopy (AFM) and Scanning Electron Microscopy (SEM), respectively. Phases can form different morphologies according to their different solubility parameters and volume fractions [37]. The corresponding geometrical relaxation of each phase has a specific lifetime significantly beyond the maximum creep time tested [36, 37].

(a)

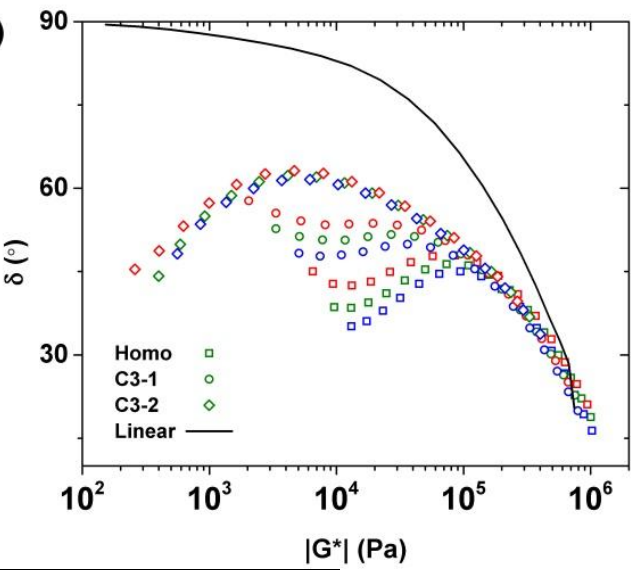

(b)

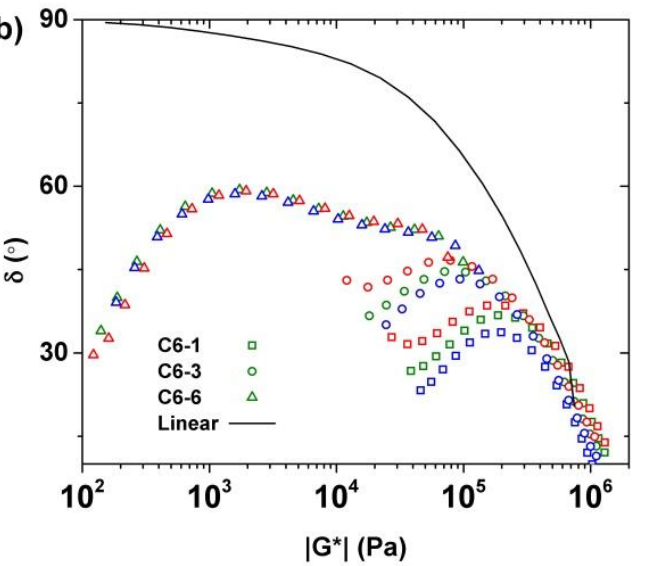

(c)

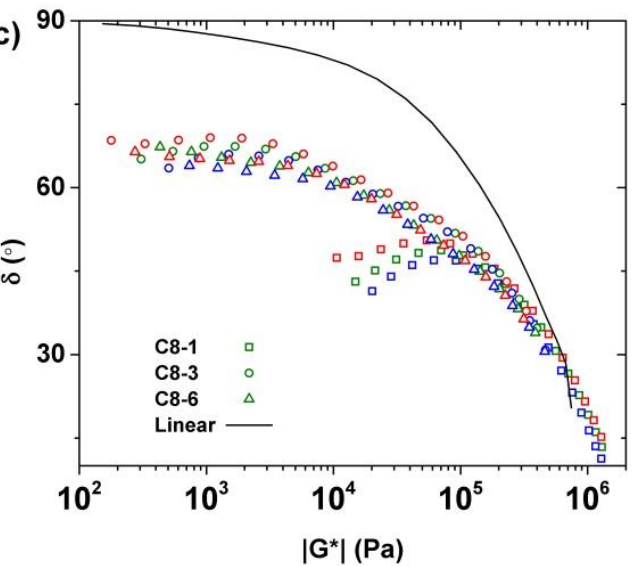


Figure 4. van Gurp-Palmen plot of (a) homopolymer and C3 (b) C6 and (c) C8 sample series, for frequency sweep measurements at 160 (blue), 190 (green) and $220^{\circ} \mathrm{C}$ (red).

Creep data were also transformed into dynamic moduli using the method of Evans et al. [38] and used to extend the frequency window towards lower values. Creep data for samples C3-2 and C8-6 were too scattered to be considered. The overlay of frequency sweep and creep data at $190{ }^{\circ} \mathrm{C}$ is shown in Figure 5. Evidently, the terminal relaxation slopes of 1 and 2 in loss and storage moduli, respectively, (unlike the behavior of the linear reference; gray lines) are not accessible even by addition of creep data. Particularly for samples with lower comonomer contents both moduli drop in parallel with the slope of 0.5 . Such behavior can be an indicator for the presence of long-chain branched chains [19, 26, 27]. Gel formation can be also made responsible for this behavior, although based on reaction kinetics and GPC-data, the former is by far the most likely possibility. It worth mentioning that degradation can be disregarded as an influence factor, as samples were stabilized with adequate amount of stabilizers and all sample preparation and rheological measurements were performed under nitrogen atmosphere. Besides for most of the samples the absence of degradation was checked by repeating the frequency sweep at $190{ }^{\circ} \mathrm{C}$ after finishing measurements at all other temperatures. In all cases, a deviation of less than $5 \%$ in $G^{\prime}(\omega)$ and G' $(\omega)$ was found between the first and second frequency sweep at $190^{\circ}$ C. For Samples C3-2 and C6-6 with higher comonomer contents, the storage moduli deviates towards a second low frequency plateau, which can be a symptom of phase separation $[36,37]$ or microgel formation. However, the presence of gel particles can be excluded from both GPC-data as well as from the reaction mechanism, which lowers LCB-content as SCB-content increase, while phase separation as an explanation fits the trend of an increasing effect with increasing SCB-content. 

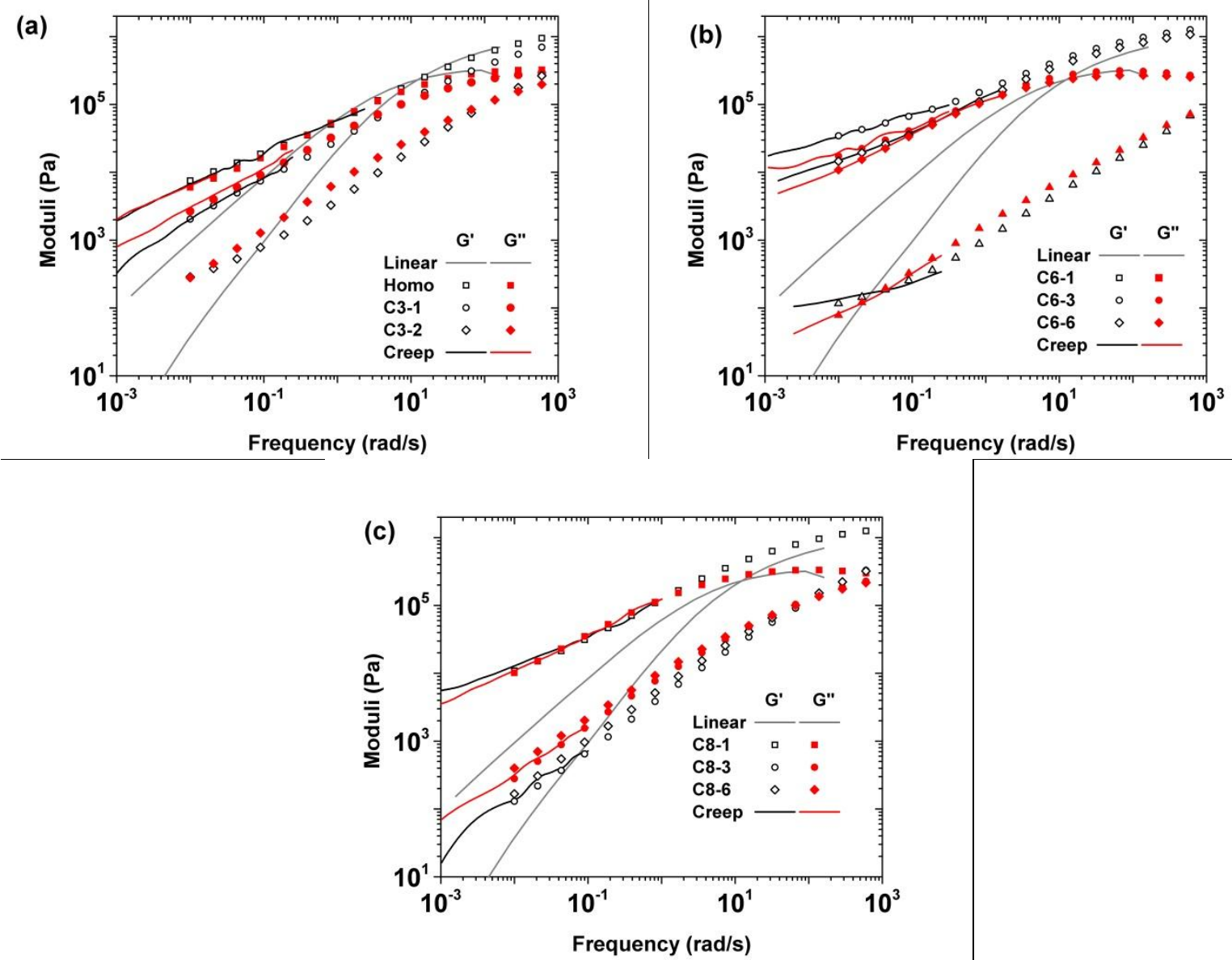

Figure 5. Dynamic moduli from overlay of frequency sweep (symbols) and creep (lines) tests at $190{ }^{\circ} \mathrm{C}$ for (a) homopolymer and C3 (b) C6 and (c) C8 sample series.

Figure 6 shows the complex viscosity of the three copolymer series along with the reference linear chain at $190{ }^{\circ} \mathrm{C}$. Clearly, the viscosity functions do not converge to a constant Newtonian viscosity at lower frequencies unlike the behavior of the linear reference sample, and instead, there is a second upturn in this region that could be due to the presence of LCB at lower comonomer contents and phase separation at higher comonomer ratios [16]. Comparing the low frequency viscosities and the molecular weight data shown in Table 1, there is a substantial deviation from the power-law dependence of zero-shear rate viscosity and weight average molecular weight as well. Both observations are consistent with the previous conclusions on complexity of the overall behavior due to microstructural diversities [19]. 

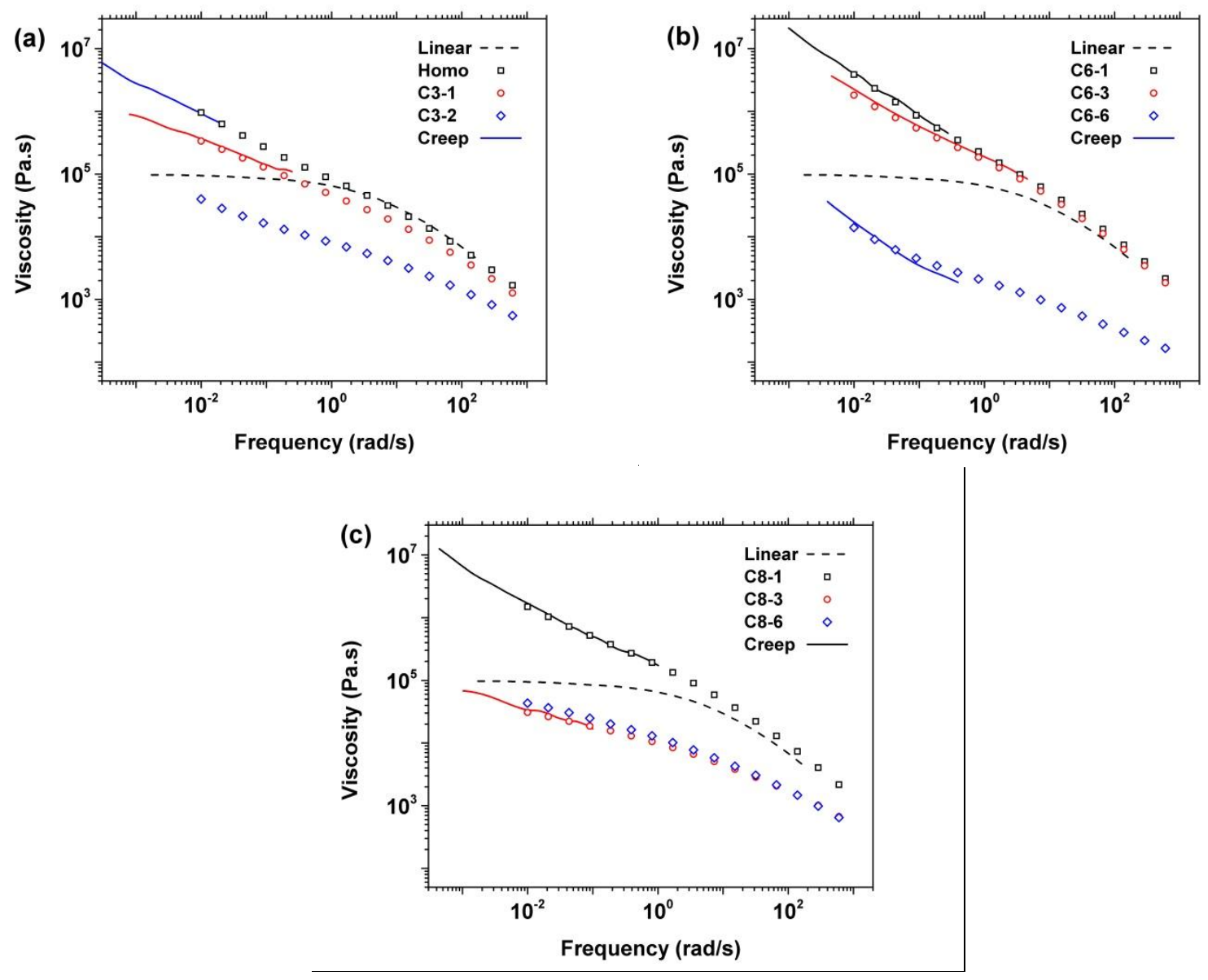

Figure 6. Complex viscosity from overlay of frequency sweep (symbols) and creep (lines) tests at $190{ }^{\circ} \mathrm{C}$ for (a) homopolymer and C3 (b) C6 and (c) C8 sample series.

The influence of the long-chain branches can be seen in a very clear fashion when picking the maximum viscosities $\eta_{\max }$ from frequency sweep data of Figure 6, i.e. at frequency of 0.01 1/s, and plotting them versus the weight average molar mass, as shown in Figure 7. The linear reference was previously established at $150^{\circ} \mathrm{C}$ [39] and shifted to $190^{\circ} \mathrm{C}$ with an activation energy $\mathrm{E}_{\mathrm{a}}$ of $28 \mathrm{~kJ} / \mathrm{mol}$, typically found for linear HDPE [40].

While it was not possible to determine the zero shear-rate viscosity $\eta_{0}$ of any of the materials within the time of stability (Figure 6), it is clear that $\eta_{0}$ is larger than the maximum viscosity measured $\eta_{\max }$. Figure 7 clearly shows that all data are located significantly above the $\eta_{0^{-}}-\mathrm{M}_{\mathrm{w}^{-}}$ 
relation for linear materials, [39] which has been established as an indicator for sparsely long-chain branched polymers, [41-43] including polyethylene [14, 44-47].

While for all samples except C3-2 and C6-6, $\eta_{\max }$ lies between $10^{6}$ and $2 \times 10^{7} \mathrm{~Pa}$.s, which is typically the maximum value one can determine for the viscosity of PE, the lower molecular C3-2 and C6-6 are relatively "low" in maximum viscosity $\left(\eta_{\max } \approx 6 \times 10^{4}\right.$ Pa.s). The application of established laws between the zero shear-rate viscosity increase factor $\eta_{0}{ }^{\mathrm{LCB}} / \eta_{0}{ }^{\mathrm{LIN}}$ is not possible, however, it can be confirmed that even samples at highest comonomer contents still possess LCB and reside above the viscosity curve for linear polyethylene.

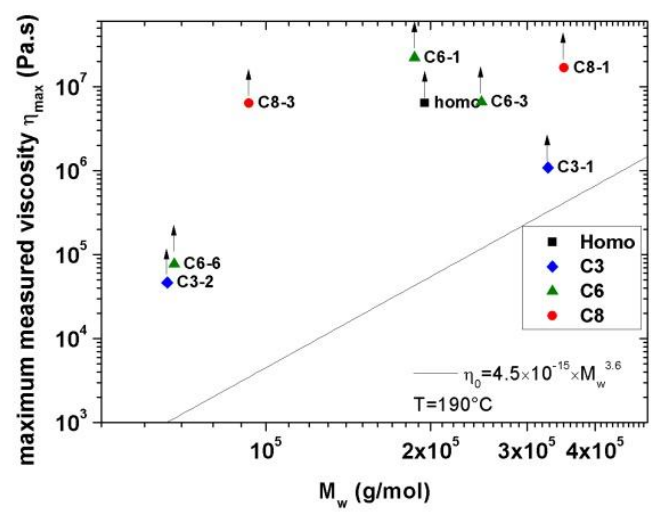

Figure 7: $\eta_{\max }-\mathrm{M}_{\mathrm{w}}$ plot at reference temperature of $190^{\circ} \mathrm{C}$.

\subsection{Insights from reaction mechanism}

Investigations of thermal and rheological properties provide very useful information about diversity of microstructural distributions in the synthesized copolymers. It was shown that samples have dual comonomer distributions, which become very pronounced at higher comonomer contents. Besides the LCB formation frequency decreases at higher comonomer contents, however a clear second plateau modulus appears at lower frequencies for samples having highest comonomer fractions, which is not thermorheologically complex and, thus, does not stem from long-chain 
branching related effects. Hence, it must originate from the significantly different comonomer contents created by the two types of active center. Hence, examination of polymerization mechanism using metallocene catalysts having oscillatory ligands can provide better insight for understanding these unique features.

Nuclear Magnetic Resonance (NMR) has been widely used to study the mechanism of olefin polymerizations by stereoselective catalysts and corresponding microstructures [21-25]. The original impression was that the unbridged metallocenes can isomerize between achiral and chiral coordination geometries during the polymerization reaction with a rate slower than monomer insertion but faster than chain formation, therefore, producing atactic-isotactic stereoblock polymers [21]. But oscillation between a rac-like (isotactic-selective) and a meso-like (nonstereoselective) conformation could not explain the observed isotactic-stereoblock polymer configuration [23]. Further investigation through computer simulations revealed that the less stable meso-like conformation should be disregarded for bulkier ligands, due to steric hindrance, and the active cation is always in rac-like conformation [48]. In other words, the active cation oscillates between the two enantiomorphous rac-like conformations, but with an average frequency that is only slightly lower than that of monomer insertion [21-23, 48]. For less bulkier ligands, like the one used in this work, the meso-like conformation becomes more stable, but not as stable as rac-like conformation, while the fast oscillation beyond the rate of monomer insertion should lead to formation of all atactic chains. However, further studies (by application of different cocatalyst and solvents) unveiled that the reversible proximity of the cocatalyst counteranion makes a transient bridge between ligands, providing temporary stereorigidity which can lead to stereoblock microstructure [23-25]. In this locked regime, the oscillation is frozen, and the active site keeps its configuration for a time long enough to produce a lengthy isotactic block. 


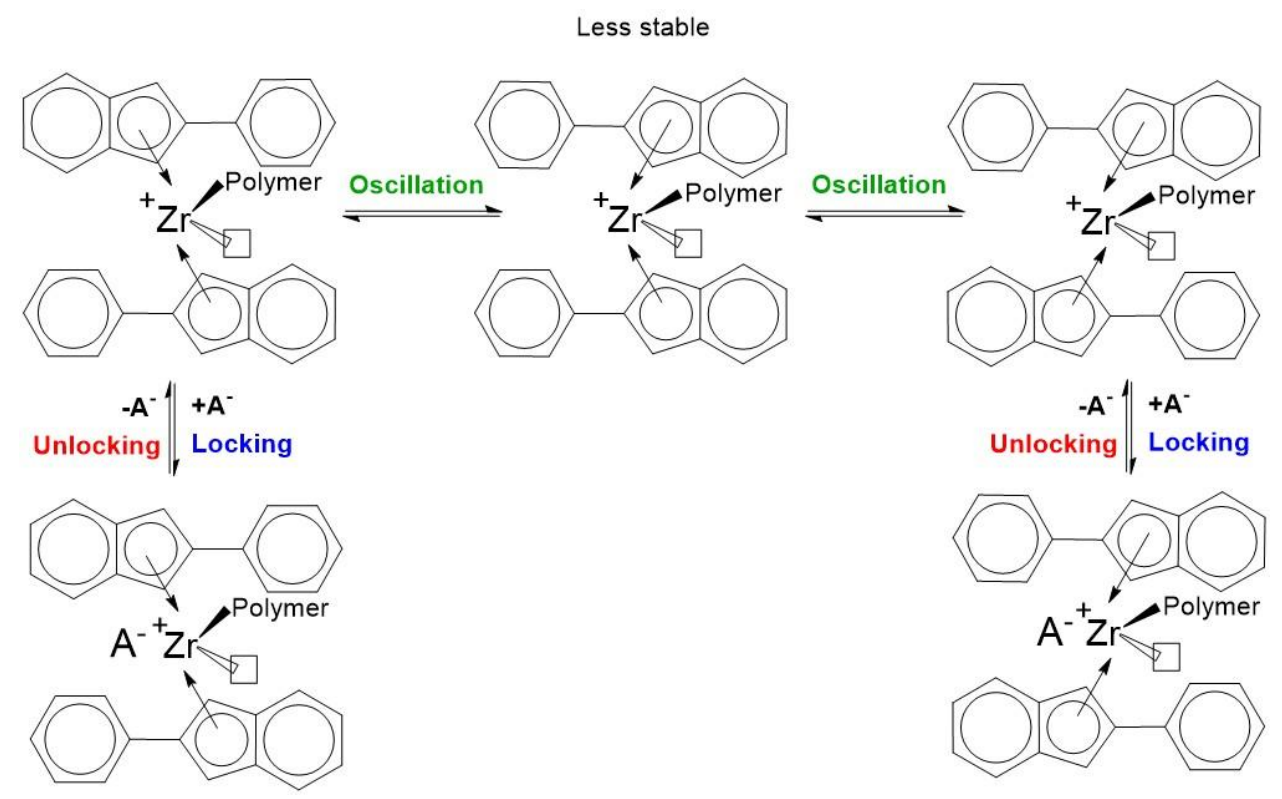

Figure 8. Oscillation of unbridged bis(2-phenylIndenyl) metallocene between two enantiomorphous racemic conformations, and reversible locking of the conformation by proximity of cocatalyst counterion.

Figure 4 illustrates the described mechanism for the utilized metallocene catalyst. The locked and the oscillating conformations should have different comonomer selectivities. The locked state is expected to have a wider face for incorporation of bulkier comonomer and in situ formed macromers (vinyl-terminated chains that are reinserted into the growing chains as long-chain branches). A 3D draw of different enantiomorphous of the catalyst and reversible locking of the conformation by proximity of cocatalyst counterion is also shown in Figure SI2 for easier comprehension of the proposed mechanism. This is apparently the source of the heterogeneities in chain compositions as depicted in MWD and DSC data. The intense difference in chemical compositions at higher comonomer fraction can even cause phase separations as confirmed by appearance of second plateau in dynamic moduli [36, 37].

The $\alpha$-olefin comonomers are prone to affect LCB formation, as they tend to terminate the growing chain by chain transfer to comonomer reaction [13]. The vinyl-terminated polymer chains are sterically hindered from incorporation as LCB due to the short-chain branches residing at the end of the growing chain and macromer. Thus, the degree of LCB tends to decrease with increasing 
comonomer content [13]. Consequently, the different comonomer response of the locked and oscillating catalyst conformations should also lead to diversity of LCB formation. The LCB formation frequency at the locked conformation with higher comonomer selectivity should decrease, while the oscillating conformation with lower comonomer affinity may not be affected, at high comonomer concentrations, as this conformation has a very low comonomer and macromer uptake anyway. Consequently, in the absence of comonomer, less branched and highly branched chains are formed by the oscillating and locked conformations, respectively. Therefore, at high comonomer fractions, almost linear chains having low and high comonomer contents will be formed by the oscillating and locked conformations, correspondingly.

\section{Conclusions}

Ethylene was copolymerized with propylene, 1-hexene, and 1-octene using the unbridged oscillatory metallocene catalyst at several comonomer feed ratios. A separate peak became distinct on the high molecular weight tail of MWD curves for samples with highest comonomer ratios that was attributed to the presence of at least two active centers with dissimilar comonomer affinities. The broad melting peak with a separate shoulder at lower melting temperatures also confirmed presence of two dissimilar comonomer affinities. Parallel drop of dynamic moduli was evident proof of LCB existence at lower comonomer values. Samples exhibited significant thermorheological complexity, whose extent decreased upon comonomer incorporation, avoiding LCB formation. Nevertheless, due to phase separation between comonomer rich and poor chains, second plateau became evident at lower frequencies, at high comonomer ratios. Furthermore, all materials contain significant but not high amounts of long-chain branches due to the shape of the viscosity function and the failure of the $\eta_{0}-\mathrm{M}_{\mathrm{w}}$-relation for linear polymers. It was concluded that the overall behavior is caused by the complex interplay of SCB and LCB. The multi-site behavior of this famous class of bis-indenyl oscillating catalyst has seemingly not been exploited by 
combination of thermal and rheological measurements. This behavior is consistent with the mechanism of fast oscillation of catalyst ligands between enantiomorphous racemic configurations, being locked upon proximity of cocatalyst counteranion, giving rise to different stereo- and comonomer selectivities.

\section{Acknowledgements}

MA would like to thank Prof. Evelyne van Ruymbeke and Prof. Christian Bailly for their continued support and hospitality beyond his postdoc in Université Catholique de Louvain, and Pascal van Velthem for his technical assistance with flash-DSC. FJS would like to thank the National Science Foundation of China (21574086), Nanshan District Key Lab for Biopolymers and Safety Evaluation (No. KC2014ZDZJ0001A), Shenzhen Sci \& Tech research grant (ZDSYS201507141105130), and Shenzhen City Science and Technology Plan Project (JCYJ20140509172719311) for financial support.

\section{References}

[1] Dankova, M., R.M. Waymouth, "High comonomer selectivity in ethylene/hexene copolymerization by unbridged indenyl metallocenes", Macromolecules 36, 3815-3820 (2003).

[2] Mortazavi, M., H. Arabi, G.H. Zohuri, S. Ahmadjo, M. Nekoomanesh, M. Ahmadi, "Ethylene Homo and Copolymerization Using a Bis - IndZrCl2 Metallocene Catalyst: Structural Composition Distribution of the Copolymer", Macromolecular Reaction Engineering 3, 263-270 (2009).

[3] Mortazavi, S.M.M., H. Arabi, G. Zohuri, S. Ahmadjo, M. Nekoomanesh, M. Ahmadi, "Copolymerization of ethylene/ a - olefins using bis (2 - phenylindenyl) zirconium dichloride metallocene catalyst: structural study of comonomer distribution", Polymer International 59, 12581265 (2010).

[4] Ahmadi, M., A. Nasresfahani, "Realistic representation of kinetics and microstructure development during chain shuttling polymerization of Olefin block copolymers", Macromolecular Theory and Simulations 24, 311-321 (2015).

[5] Ahmadi, M., M.R. Saeb, Y. Mohammadi, M.M. Khorasani, F.J. Stadler, "A perspective on modeling and characterization of transformations in the blocky nature of olefin block copolymers", Industrial \& Engineering Chemistry Research 54, 8867-8873 (2015).

[6] Arriola, D.J., E.M. Carnahan, P.D. Hustad, R.L. Kuhlman, T.T. Wenzel, "Catalytic production of olefin block copolymers via chain shuttling polymerization", Science 312, 714-719 (2006). 
[7] Khorasani, M.M., M.R. Saeb, Y. Mohammadi, M. Ahmadi, "The evolutionary development of chain microstructure during tandem polymerization of ethylene: A Monte Carlo simulation study", Chemical Engineering Science 111, 211-219 (2014).

[8] Mohammadi, Y., M. Ahmadi, M.R. Saeb, M.M. Khorasani, P. Yang, F.J. Stadler, "A detailed model on kinetics and microstructure evolution during copolymerization of ethylene and 1-octene: from coordinative chain transfer to chain shuttling polymerization", Macromolecules 47, 4778-4789 (2014). [9] Wang, W., Z.-q. Fan, L.-x. Feng, C.-h. Li, "Substituent effect of bisindenyl zirconene catalyst on ethylene/1-hexene copolymerization and propylene polymerization", European polymer journal 41, 8389 (2005).

[10] Kaminsky, W., C. Piel, K. Scharlach, Polymerization of ethene and longer chained olefins by metallocene catalysis, Macromolecular Symposia, Wiley Online Library, 2005, pp. 25-34.

[11] Karimkhani, V., F. Afshar-Taromi, S. Pourmahdian, F.J. Stadler, "Revisiting the long-chain branch formation mechanism in metallocene catalyzed polyethylenes", Polymer Chemistry 4, 3774-3790 (2013).

[12] Münstedt, H., T. Steffl, A. Malmberg, "Correlation between rheological behaviour in uniaxial elongation and film blowing properties of various polyethylenes", Rheologica acta 45, 14-22 (2005). [13] Stadler, F.J., C. Piel, K. Klimke, J. Kaschta, M. Parkinson, M. Wilhelm, W. Kaminsky, H. Münstedt, "Influence of type and content of various comonomers on long-chain branching of ethene/ $\alpha$-olefin copolymers", Macromolecules 39, 1474-1482 (2006).

[14] Stadler, F.J., V. Karimkhani, "Correlations between the characteristic rheological quantities and molecular structure of long-chain branched metallocene catalyzed polyethylenes", Macromolecules 44, 5401-5413 (2011).

[15] Stadler, F.J., J. Kaschta, H. Münstedt, "Thermorheological behavior of various long-chain branched polyethylenes", Macromolecules 41, 1328-1333 (2008).

[16] Stadler, F.J., H. Münstedt, "Correlations between the Shape of Viscosity Functions and the Molecular Structure of Long - Chain Branched Polyethylenes", Macromolecular Materials and Engineering 294, 25-34 (2009).

[17] Robertson, C.G., C.A. García - Franco, S. Srinivas, "Extent of branching from linear viscoelasticity of long - chain - branched polymers", Journal of Polymer Science Part B: Polymer Physics 42, 1671-1684 (2004).

[18] Takeh, A., J. Worch, S. Shanbhag, "Analytical rheology of metallocene-catalyzed polyethylenes", Macromolecules 44, 3656-3665 (2011).

[19] van Ruymbeke, E., S. Coppola, L. Balacca, S. Righi, D. Vlassopoulos, "Decoding the viscoelastic response of polydisperse star/linear polymer blends", Journal of Rheology (1978-present) 54, 507-538 (2010).

[20] Wiyatno, W., Z.-R. Chen, Y. Liu, R.M. Waymouth, V. Krukonis, K. Brennan, "Heterogeneous composition and microstructure of elastomeric polypropylene from a sterically hindered 2arylindenylhafnium catalyst", Macromolecules 37, 701-708 (2004).

[21] Coates, G.W., R.M. Waymouth, "Oscillating stereocontrol: a strategy for the synthesis of thermoplastic elastomeric polypropylene", Science 267, 217 (1995).

[22] Lin, S., R.M. Waymouth, "2-arylindene metallocenes: Conformationally dynamic catalysts to control the structure and properties of polypropylenes", Accounts of chemical research 35, 765-773 (2002).

[23] Busico, V., V. Van Axel Castelli, P. Aprea, R. Cipullo, A. Segre, G. Talarico, M. Vacatello, "“Oscillating” Metallocene Catalysts: What Stops the Oscillation?", Journal of the American Chemical Society 125, 5451-5460 (2003).

[24] Nejabat, G.R., M. Nekoomanesh, H. Arabi, H. Salehi - Mobarakeh, G.H. Zohuri, M. Omidvar, S.A. Miller, "Synthesis and microstructural study of stereoblock elastomeric polypropylenes from 
metallocene catalyst (2 - Phlnd) $2 \mathrm{ZrCl} 2$ activated with cocatalyst mixtures", Journal of Polymer Science Part A: Polymer Chemistry 51, 724-731 (2013).

[25] Wilmes, G.M., J.L. Polse, R.M. Waymouth, "Influence of cocatalyst on the stereoselectivity of unbridged 2-phenylindenyl metallocene catalysts", Macromolecules 35, 6766-6772 (2002).

[26] Ahmadi, M., C. Bailly, R. Keunings, M. Nekoomanesh, H. Arabi, E. Van Ruymbeke, "Time marching algorithm for predicting the linear rheology of monodisperse comb polymer melts", Macromolecules 44, 647-659 (2011).

[27] Liu, J., W. Yu, C. Zhou, "Polymer chain topological map as determined by linear viscoelasticity", Journal of Rheology (1978-present) 55, 545-570 (2011).

[28] Mortazavi, S.M.M., H. Jafarian, M. Ahmadi, S. Ahmadjo, "Characteristics of linear/branched polyethylene reactor blends synthesized by metallocene/late transitional metal hybrid catalysts", Journal of Thermal Analysis and Calorimetry 123, 1469-1478 (2016).

[29] Awudza, J.A., P.J. Tait, "The "comonomer effect" in ethylene/ a - olefin copolymerization using homogeneous and silica - supported $\mathrm{Cp} 2 \mathrm{ZrCl} 2 / \mathrm{MAO}$ catalyst systems: Some insights from the kinetics of polymerization, active center studies, and polymerization temperature", Journal of Polymer Science Part A: Polymer Chemistry 46, 267-277 (2008).

[30] Kissin, Y.V., "Main kinetic features of ethylene polymerization reactions with heterogeneous Ziegler-Natta catalysts in the light of a multicenter reaction mechanism", Journal of Polymer Science Part A: Polymer Chemistry 39, 1681-1695 (2001).

[31] Kissin, Y.V., "Active centers in Ziegler-Natta catalysts: Formation kinetics and structure", Journal of catalysis 292, 188-200 (2012).

[32] Hosoda, S., "Structural distribution of linear low-density polyethylenes", Polym. J. 20, 383-397 (1988).

[33] van Drongelen, M., P. Roozemond, E. Troisi, A. Doufas, G. Peters, "Characterization of the primary and secondary crystallization kinetics of a linear low-density polyethylene in quiescent-and flowconditions", Polymer 76, 254-270 (2015).

[34] Saeb, M.R., Y. Mohammadi, M. Ahmadi, M.M. Khorasani, F.J. Stadler, "A Monte Carlo-based feeding policy for tailoring microstructure of copolymer chains: Reconsidering the conventional metallocene catalyzed polymerization of $\alpha$-olefins", Chemical Engineering Journal 274, 169-180 (2015).

[35] Ahmadi, M., S.M.M. Mortazavi, S. Ahmadjo, M. Zahmati, K. Valieghbal, D. Jafarifar, R. Rashedi, "Evaluation of continuous and discrete melting endotherms in determination of structural heterogeneities in Ziegler-Natta catalyzed linear low density polyethylene", Polyolefins Journal 3, 135146 (2016).

[36] Lohse, D.J., "The influence of chemical structure on polyolefin melt rheology and miscibility", Journal of Macromolecular Science, Part C: Polymer Reviews 45, 289-308 (2005).

[37] Kossuth, M., D. Morse, F. Bates, "Viscoelastic behavior of cubic phases in block copolymer melts", Journal of Rheology (1978-present) 43, 167-196 (1999).

[38] Evans, R., M. Tassieri, D. Auhl, T.A. Waigh, "Direct conversion of rheological compliance measurements into storage and loss moduli", Physical Review E 80, 012501 (2009).

[39] Stadler, F.J., C. Piel, J. Kaschta, S. Rulhoff, W. Kaminsky, H. Münstedt, "Dependence of the zero shear-rate viscosity and the viscosity function of linear high-density polyethylenes on the mass-average molar mass and polydispersity", Rheologica Acta 45, 755-764 (2006).

[40] Stadler, F.J., C. Gabriel, H. Münstedt, "Influence of Short - Chain Branching of Polyethylenes on the Temperature Dependence of Rheological Properties in Shear", Macromolecular Chemistry and Physics 208, 2449-2454 (2007).

[41] Auhl, D., J. Stange, H. Münstedt, B. Krause, D. Voigt, A. Lederer, U. Lappan, K. Lunkwitz, "Long-chain branched polypropylenes by electron beam irradiation and their rheological properties",

Macromolecules 37, 9465-9472 (2004). 
[42] Bersted, B., "On the effects of very low levels of long chain branching on rheological behavior in polyethylene", Journal of applied polymer science 30, 3751-3765 (1985).

[43] Bersted, B., J. Slee, C. Richter, "Prediction of rheological behavior of branched polyethylene from molecular structure", Journal of Applied Polymer Science 26, 1001-1014 (1981).

[44] Gabriel, C., Einfluss der molekularen Struktur auf das viskoelastische Verhalten von Polyethylenschmelzen, Shaker2001.

[45] Janzen, J., R. Colby, "Diagnosing long-chain branching in polyethylenes", Journal of Molecular Structure 485, 569-584 (1999).

[46] Piel, C., F.J. Stadler, J. Kaschta, S. Rulhoff, H. Münstedt, W. Kaminsky, "Structure - Property Relationships of Linear and Long - Chain Branched Metallocene High - Density Polyethylenes

Characterized by Shear Rheology and SEC - MALLS", Macromolecular Chemistry and Physics 207, 26-38 (2006).

[47] Wood-Adams, P., The effect of long chain branching on the rheological behavior of polyethylenes synthesized using constrained geometry and metallocene catalysts, 1998.

[48] Wilmes, G.M., M.B. France, S.R. Lynch, R.M. Waymouth, "Effects of ligand substitutions on the rotation rate of indenyl ligands in bis (2-arylindenyl) zirconocenes by NMR line-shape analysis and relaxation in the rotating frame", Organometallics 23, 2405-2411 (2004). 


\section{FOR TOC-use only}

Complex interplay of short and long-chain branching on thermal and rheological properties of ethylene/ $\alpha$-olefin copolymers made by oscillatory metallocene catalyst

Mostafa Ahmadi, Faeze Rezaei, Mohammad Mehdi Mortazavi, Mehdi Entezam, Florian J. Stadler

Oscillating metallocene catalyst is used in copolymerization of ethylene and several $\alpha$-olefin comonomers. The dual nature of active centers leading to complex interplay of SCB and LCB is revealed by studying thermal and rheological properties.

\section{Low Comonomer High Comonomer}
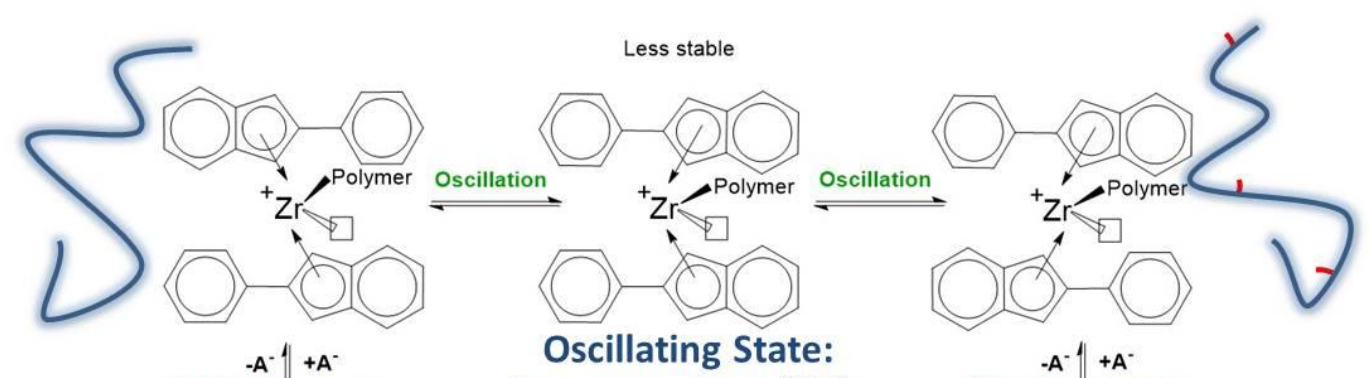

Unlocking Locking Lower comonomer affinity

$-A^{-1}+A^{-}$

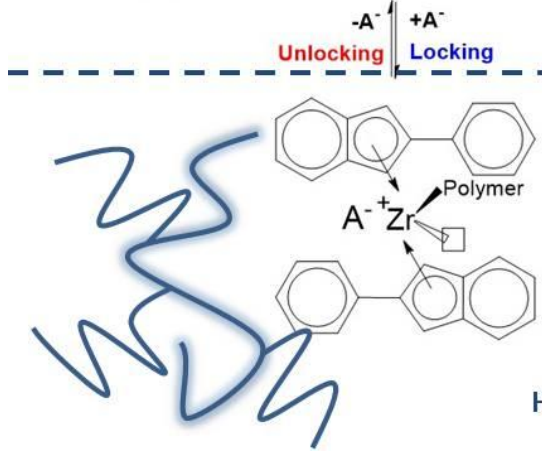

Locked State:

Higher comonomer \& macromer affinity

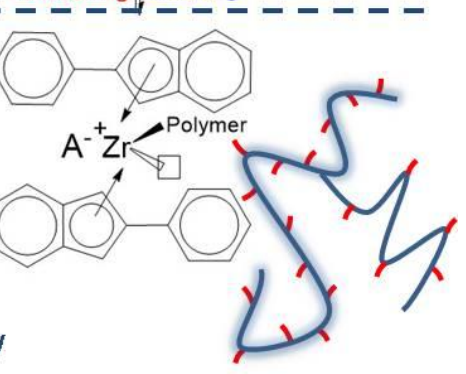




\section{Graphical Abstract}

Complex interplay of short and long-chain branching on thermal and rheological properties of ethylene/ $\alpha$-olefin copolymers made by oscillatory metallocene catalyst

Mostafa Ahmadi, Faeze Rezaei, Mohammad Mehdi Mortazavi, Mehdi Entezam, Florian J. Stadler

Oscillating metallocene catalyst is used in copolymerization of ethylene and several $\alpha$-olefin comonomers. The dual nature of active centers leading to complex interplay of SCB and LCB is revealed by studying thermal and rheological properties.

\section{Low Comonomer High Comonomer}

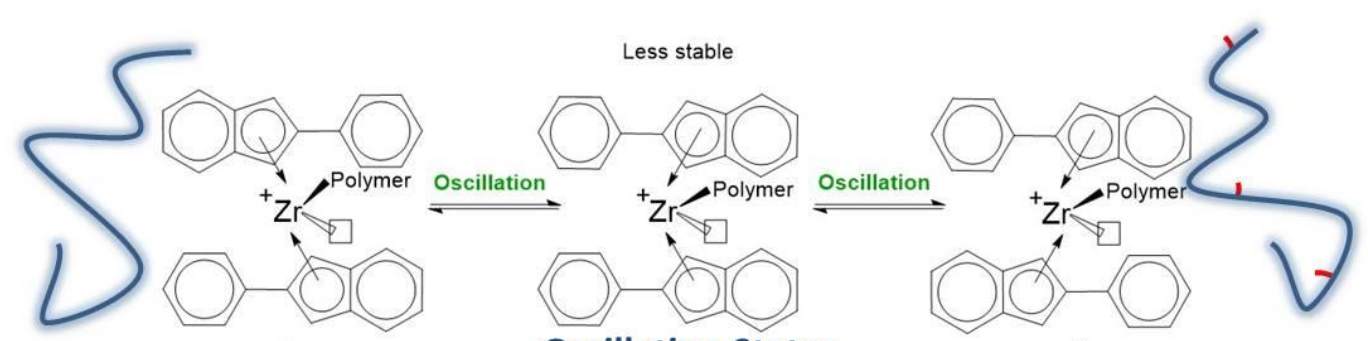

$-A^{-4}+A^{-} \quad$ Oscillating State:

Unlocking Locking $\quad$ Lower comonomer affinity

$-A^{-1}+A^{-1}$

Unlocking, Locking

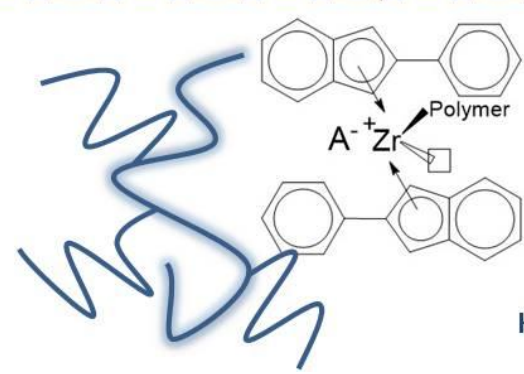

Locked State:

Higher comonomer \& macromer affinity

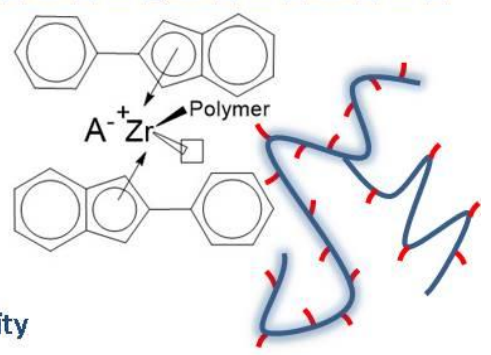

